\section{DIGITAL COMMONS \\ @ UNIVERSITY OF SOUTH FLORIDA}

Journal of African Conflicts and Peace Studies

Volume 2

Issue 1 Special Double Issue: Democratization

Process in the Great Lakes Region: Progress

Article 8 and Challenges

September 2013

\title{
Egalite du Genre Dans le Processus de la Stabilisation de la S/ Region des Grands Lacs : Quels Enjeux Dans le Processus Electoral 2011 en RDC?
}

Vincent Muderhwa Barhatulirwa

mmuderwa@yahoo.fr

Follow this and additional works at: https://digitalcommons.usf.edu/jacaps

Part of the Social and Behavioral Sciences Commons

\section{Recommended Citation}

Barhatulirwa, Vincent Muderhwa (2013) "Egalite du Genre Dans le Processus de la Stabilisation de la S/ Region des Grands Lacs : Quels Enjeux Dans le Processus Electoral 2011 en RDC?," Journal of African Conflicts and Peace Studies: Vol. 2: Iss. 1, 83-95.

DOI: http://dx.doi.org/10.5038/2325-484X.2.1.8

Available at: https://digitalcommons.usf.edu/jacaps/vol2/iss1/8

This Article is brought to you for free and open access by the Open Access Journals at Digital Commons @ University of South Florida. It has been accepted for inclusion in Journal of African Conflicts and Peace Studies by an authorized editor of Digital Commons @ University of South Florida. For more information, please contact digitalcommons@usf.edu. 


\title{
EGALITE DU GENRE DANS LE PROCESSUS DE LA STABILISATION DE LA S/REGION DES GRANDS LACS : quels enjeux dans le processus électoral 2011 en RDC ?
}

\author{
Prof Vincent MUDERHWA BARHATULIRWA ${ }^{1}$
}

\begin{abstract}
Absract: La question du genre m'intéresse car 'l'institutionnalisation sociale des différences des sexes va au cœur du politique, et donc au cœur de la paix'. La stabilité socio-économique et politique de la Sous-Région dépendra de la promotion du genre. Le point de départ de cette réflexion consistera à faire une analyse du contexte. D'abord, faire découvrir que la plupart des Etats fonctionnent comme un système social patriarcal imbu des cultures et des traditions marginalisant les femmes par des formes de langage qui les dévalorisant et les décourageant à jouer un rôle dans le domaine public. Ensuite, essayer de comprendre comment les drames dus à la guerre donnant des statistiques effrayantes des viols et violences faites aux femmes ont malheureusement impliqué un discours de victimisation des femmes. Et la conséquence, c'est l'état de passivité dans lequel les femmes sont maintenues, parce que définies comme ayant des besoins spéciaux de protection et déterminées à n'être que mère et bonne femme. D'où penser stabiliser la Région, c'est aussi s'engager pour la promotion de l'égalité du genre. Que faire? (i) s'engager dans la dynamique de la transformation de la société ; ce qui exige un travail d'éducation de toutes les couches à adopter et intérioriser une vision moderne du genre; (ii) opérer des réformes structurelles par des 'affirmative actions' consistant à promouvoir l'égalité intra et/ou inter groupes sur le plan institutionnel en introduisant, par exemple, le système des quotas en vue d'une représentation proportionnelle des hommes et des femmes dans les espaces de décision à tous les niveaux; (iii) associer les femmes comme actrices et non observatrices à la table de négociation de la paix.
\end{abstract}

\section{INTRODUCTION}

La question du genre est régulièrement abordée par les femmes comme si elles étaient les seules concernées par ce débat. La dernière décennie du $20^{\text {ème }}$ siècle a été marquée par des conflits armés de grande intensité qui ont désorganisé plusieurs Etats de l'Afrique des Grands Lacs. D'une part, les guerres ont fait sombrer le Burundi et le Rwanda dans des conflits intercommunautaires. Les conflits qui ont secoué ces pays se sont propagés comme par un mécanisme des vases communicants à la RDC qui s'est enlisée, à son tour dans un conflit dans lequel plusieurs armées des pays voisins (Rwanda, Burundi, Uganda, Angola, Zimbabwe, etc) et plusieurs factions et milices rivales s'affrontèrent sur son territoire. Les femmes ont souvent été les cibles directes des conflits dont elles ne sont ni les instigatrices ni les protagonistes par des viols et violences dont elles ont été victimes.

\section{Directeur du CAREPD/ULPGL-Goma}


Les enquêtes qui ont été menées ont joué un rôle non négligeable dans les efforts d'établir les faits tels qu'ils ont été vécus. Cependant, le langage victimisant les femmes a malheureusement été exploité autrement. Les femmes sont considérées par les hommes comme faibles, vulnérables et incapables d'articuler leurs propres besoins. Il va sans dire qu'elles se considèrent comme telles. D'où elles se sont laissé emporter dans l'empathie et la passivité croyant qu'elles ne peuvent que compter sur les hommes pour les protéger.

Etablir des statistiques n'est pas mauvais en soi mais s'attarder à victimiser les femmes pose problème. La stratégie ne devrait-elle pas être orientée vers la nécessité d'engager tous les groupes sociaux dans la dynamique de la construction de la paix ? Dans une période postconflit, il me semble, promouvoir le genre dans toutes les structures sociales (famille, école, université, milieux professionnels, église, et surtout dans l'espace politique, etc) pourrait être le socle stratégique dans le processus de la stabilisation de la Sous-Région des Grands Lacs. Les femmes qui ont longtemps porté le poids de la discrimination, des injustices et des violences dans plusieurs structures sociales et surtout dans la sphère politique, devraient être assis sur la table de négociation de la paix à égalité des voix avec leurs bourreaux qui sont majoritairement les hommes. On ne peut penser paix sans qu'il y ait un effort des uns et des autres de bannir toutes les formes de discrimination liées aux pesanteurs traditionnelles, culturelles et à des perceptions fixistes qui méconnaissent à la femme les mêmes droits et mêmes avantages autant qu'à l'homme.

Nous allons, de prime abord, essayer de comprendre les concepts « genre » et «égalité/équité du genre », avant de faire une analyse du contexte qui nous permettra de comprendre comment les traditions patriarcales ont engendré les discriminations et les inégalités. En troisième lieu, nous nous efforcerons de recenser quelques obstacles structurels relatifs au genre qui jalonnent le parcours de la recherche de la paix. Après avoir inventorié les obstacles, nous montrerons à quel niveau les inégalités du genre est un grand frein au développement. Nous terminerons par les voies et moyens qu'il conviendrait d'utiliser pour promouvoir l'égalité du genre en dégageant les avantages insoupçonnés de cette stratégie.

\section{DEFINITION DES CONCEPTS}

\subsection{Le concept « Genre »}

Le genre est un concept se référant aux différences sociales entre les femmes et les hommes. L'analyse du concept genre se concentre sur les relations entre les hommes et les femmes et sur les situations, souvent inégalitaires, qui découlent de ces relations. Le genre fait référence à la construction sociale et culturelle des identités femelles et males. Les constructions sociales et culturelles au sujet des hommes et des femmes conduisent à des rôles socialement et culturellement construits. Les perceptions différentiées et les attentes des hommes et des femmes les conduisent à occuper des positions spécifiques et/ou un espace dans la société. Les différences qui proviennent de ces constructions sociales, attitudes, perceptions et attentes changent au fil du temps et varient dans et parmi les cultures et même dans les contextes 
politiques et économiques ${ }^{2}$. Elles varient tant à l'intérieur des groupes que selon les différentes cultures ${ }^{3}$ et traditions.

\subsection{Le concept « égalité »}

Le terme "égalité » est conceptualisé à partir d'une approche basée sur des droits selon laquelle tous les êtres humains sont libres de développer leurs habilités personnelles et faire des choix sans limitations placées par les rôles standards du genre. D'une part, l'égalité du genre est une considération et la valorisation des aspirations différentes, des préoccupations et des besoins des hommes et des femmes. Le souci est de donner des droits égaux et offire les mêmes opportunités aux hommes et aux femmes, aux garçons et aux filles de participer pleinement dans les sphères sociales/culturelles, économiques et politiques en vue de promouvoir un développement durable ${ }^{4}$. D'autre part, l'équité du genre se réfère à la justice, à l'impartialité, dans le traitement et la reconnaissance du potentiel et l'accès à l'opportunité à la fois aux hommes et aux femmes. Il s'agit de l'application de la justice et impartialité dans l'allocation des ressources, services et bénéfices entre hommes et femmes. Les deux sexes ont conséquemment à condition égale les mêmes droits et avantages en vue de réaliser leur potentiel et contribuer, pour ainsi dire, au développement durable. Dans ce sens, le combat pour l'égalité et l'équité du genre n'annihile ni ne méconnait les différences qui existent entre l'homme et la femme. Les différences ne sont pas perçues comme un obstacle mais un enrichissement mutuel. Elles sont abordées, avec un sens d'équité, en vue de rectifier les inégalités et les déséquilibres parce que tout ce qui tend à désavantager les femmes au profit des hommes porte atteinte au développement. Dès lors que nous comprenons déjà les concepts et expressions utilisés, nous pouvons dire un mot sur le contexte tel qu'il se présente en rapport avec le genre.

\section{III.ANALYSE DU CONTEXTE}

Les inégalités et les discriminations entre les hommes et les femmes peuvent être comptées parmi les antivaleurs qui rongent encore notre société. Les inégalités persistent en termes d'accès à l'éducation, à la santé, à la justice, à l'emploi mais aussi en terme d'accès à certaines positions tant en politique qu'en administration, positions dominées à $90 \%$ par les hommes, etc. La stratégie nationale de la lutte contre les violences basées sur le Genre en RDCongo le dit en ces termes :

L'analyse du Genre met en évidence la persistance des déséquilibres en matière de parité entre hommes et femmes dans tous les domaines de développement: économiques, sociaux, culturels et politiques. Ces déséquilibres constituent des obstacles à la jouissance

\footnotetext{
${ }^{2}$ C. Kabonesa, Gender Mainstreaming and Implications for Peace. In: D. Rodriguez \& E. Natukunda-Togboa, Gender and Peacebuilding, San José: University for Peace, p.13. ${ }^{3}$ B. Hounton, "Genre et droits de l'homme". In : Actes du séminaire de Formation sur le Genre, les droits de l'homme et la démocratie, Kisangani, 16-21 mars 2004, p.17.

${ }^{4}$ C. Kabonesa, op.cit., p.15.

${ }^{5}$ Ibid., p.15.
} 
des mêmes droits humains pour tous et empêchent ainsi les femmes de jouer pleinement leur rôle dans le processus de développement du pays ${ }^{6}$.

Les inégalités observées dans l'éducation sont telles que le taux de scolarisation des filles est inférieur à celui des garçons tandis que le taux de déperdition scolaire et même celui de l'analphabétisme est plus important que celui des garçons. L'analphabétisme touche un grand nombre de femmes et cela surtout en milieu rural; une femme sur deux est analphabète curieusement contre un homme sur cinq. Les inégalités dans le travail sont réelles au regard de ce pourcentage : $70 \%$ des femmes se concentrent dans l'agriculture traditionnelle et $60 \%$ dans le secteur informel notamment le petit commerce des denrées alimentaires (tomates, bananes, poissons, pommes de terre, riz, sucre, etc). Les deux secteurs constituent le cœur de l'économie réelle qui fait survivre la majorité des familles et contribuent à la scolarisation depuis plus de deux décennies à la suite de quasi-absence et/ou de la modicité des salaires dans le secteur de la Fonction Publique.

Analysant le contexte, on retient que les traditions patriarcales ont fait tâche d'huile dans nos Etats pour une raison majeure. La perception différenciée des rôles masculins et féminins et des relations familiales entre les hommes et les femmes ont entrainé l'infériorisation de la jeune-fille et la femme. Malgré la diversité des croyances religieuses et pratiques culturelles, la majorité des groupes ethniques et sociaux, que ce soit en RDCongo, au Rwanda, au Burundi ou ailleurs, ont en commun la même perception différenciée des rôles masculins et féminins et des relations familiales entre les hommes et les femmes sont construits sur l'inégalité fondamentale entre l'homme et la femme'. L'homme est perçu comme l'époux, le chef de famille, le gestionnaire du foyer, celui qui incarne l'autorité au sein du ménage, qui fixe les règles et le code de conduite, assure le contrôle et la gestion des biens familiaux. La femme, quant à elle, est réduite au rôle de la mère et épouse, à la charge sociale du fonctionnement de la vie domestique. Son rôle, c'est celui de réaliser les travaux ménagers, prendre soin des enfants et autres membres de la famille. La division sexiste du travail et des rôles à jouer déterminent la répartition des tâches entre les filles et les garçons dès le bas âge en famille. Cette différenciation assigne aux hommes des travaux de production formels et qui sont de loin valorisés que ceux des femmes assignées à des activités de reproduction non valorisées $^{8}$. L'éducation sexuée, familiale et scolaire encourage les filles à adopter des attitudes qui sont conformes à leurs sexes et qui a des retombées dans la vie future. Une telle éducation influence les choix de loisirs, de métiers et génère, pour ainsi dire, des inégalités dont les principales victimes sont les femmes.

Dans ce sens, la culture demeure le plus grand obstacle au traitement équitable des femmes. Le titre intriguant de l'étude de Sylvia Tamale «When Hens Begin to Crow » qui fonde son origine dans les élections générales de l'Uganda en 1996 révèle le langage méprisant tenu à l'égard d'une femme candidate à ces élections :

\footnotetext{
${ }^{6} \mathrm{Cf}$. www.observatoire parité/rdcongo, campagne pour l'égalité filles/garçons, Tugawe, p.1.

${ }^{7} \mathrm{Cf}$. www.observatoire parité/rdcongo, campagne pour l'égalité filles/garçons, Tugawe, p.2.

${ }^{8}$ Ibid., p.3.
} 
Female chickens do not normally crow. At least popular mythology claims that they cannot. Hence, in many African cultures, a crowing hen is considered as an omen of bad tidings that must be expiated through the immediate slaughter of the offending bird. The message was clear: Women have no business standing for political office ${ }^{9}$.

La considération de la femme comme une poule non autorisée à chanter comme le fait le coq au village appliquée à la sphère politique est non seulement une aberration mais aussi une évidence que dans les structures patriarcales existantes dans la Sous-Région, la politique n'est pas une affaire des femmes. Cela se vérifie dans la manière dont les femmes sont considérées dans les milieux politiques où elles paient les frais des harcèlements sexuels de la part de leurs collègues hommes et sont couvertes de ridicule dans les débats. On pourrait croire que leur présence dans l'espace politique n'est qu'un symbole sans valeur réelle pour le développement de la Nation. Voilà qui fait piétiner le processus de la construction de la paix et du développement.

\section{IV.QUELQUES OBSTACLES STRUCTURELS A LA CONSTRUCTION DE LA PAIX ET CONSEQUENCES DES INEGALITES DU GENRE}

\subsection{Des obstacles structurels}

Dans son article, Nadine Puechguilbal fait remarquer qu'il existe des obstacles qui s'abattent dangereusement sur le processus de la construction de la paix en Afrique. Pour elle, le langage utilisé dans la perception de la femme est l'un des obstacles majeurs dans la dynamique de la construction de la paix. Elle est la clé indicatrice de la perception faite sur l'homme et la femme dans une société donnée et quels rôles ils sont appelés à jouer. ${ }^{10}$ La thèse que soutient Nadine, dans son article intitulé «Gender and Peace Building in Africa: Analysis of some Structural Obstacles », c'est que tant que les femmes sont confinées à leur destin biologique dans le domaine privé, elles ne seront jamais acceptés comme individus autonomes dans l'arène publique ${ }^{11}$. Le point de départ de sa réflexion, c'est l'examen du langage sensible du genre dans les Accords de paix et les résolutions des Nations Unies. L'idée qui traverse en filigrane toutes les résolutions de l'ONU et les accords de paix, c'est que les femmes sont principalement définies comme victimes; ce qui limite leur capacité d'être considérées comme actrices et individus indépendants dans le processus de paix ${ }^{12}$. En parcourant les accords de paix à Lomé en rapport avec le conflit en Sierra Léone, les femmes sont vues comme gardiennes de la base morale de la société, de l'honneur de la famille et elles sont porteuses des valeurs culturelles de la communauté. Dans ce sens, les efforts sont concentrés sur la protection des femmes et des enfants. Au Liberia, l'accord de paix ne contient aucune

\footnotetext{
${ }^{9}$ A Ruhangataremwa, cité par Fatoumatta M'boge \& S. Gbaydee Doe, African Commitments to Civil Society Engagement. A Review of Eight NEPAD Countries. African Human Security Initiative, 2004, p.85.

${ }^{10}$ Nadine Puechguirbal, 'Gender and Peacebuilding in Africa: Analysis of Some Structural Obstacles'. San José: University for Peace, p.5.

11 Ibid., p.2.

12 Ibid., p.2.
} 
perspective du genre. Les femmes constituent une minorité sociologique au même titre que les catégories basées sur l'âge, la couleur, la religion, l'handicap, le groupe ethnique. Dans le cas de la Côte d'Ivoire, l'accord de paix ne contient aucun langage sensible du genre en dépit du fait qu'il fait mention des questions de la sécurité, de violation des droits humains, de l'assistance humanitaire des populations comme si hommes et femmes, garçons et filles avaient les mêmes besoins et priorités et comme si tous ces groupes sociaux font mécaniquement la même expérience de conflit en Côte d'Ivoire.

En outre, Nadine va plus loin en disant que «the use of a language that defines women first and foremost in their reproductive role participates in making them more vulnerable and somehow jeopardizes their lives by anchoring a conservative vision of gender roles in the mind of the people $»{ }^{13}$ Le fait de définir exclusivement les femmes dans leur rôle de reproduction ne fait que les appréhender comme être humains faibles ayant besoin d'être protégés et n'ayant pas de contrôle sur leurs propres vies. Définir les femmes par rapport à ce qu'elles sont et non ce qu'elles font n'est rien d'autre que «l'anatomie du destin » des femmes, dit la philosophe française Simone de Beauvoir. Lorsque les femmes sont souvent associées à leur capacité maternelle seulement, comme conséquence, elles sont construites comme des éléments passifs subordonnés au pouvoir du male et elles sont exclues des activités politiques et des négociations officielles de paix. Une telle perception des femmes entraine des conséquences énormes.

\subsection{Des conséquences des inégalités du genre}

Une étude élaborée par une corporation autrichienne de développement en Ethiopie a fait observer que les inégalités du genre imposent un large coût sur la santé et le bien-être des hommes, des femmes et des enfants, et affectent leur habilité d'améliorer leurs conditions de $v_{i e}{ }^{14}$. L'étude révèle que les inégalités du genre réduisent la productivité et donc exacerbent la pauvreté.

Une autre conséquence de ces inégalités, c'est l'affaiblissement de la gouvernance du pays et donc l'effectivité de ses politiques de développement. Ce qui, du reste, est évident dans tous les pays du monde, c'est que les sociétés avec larges et persistantes inégalités du genre payent le prix de plus de pauvreté, de malnutrition, de maladie, et autres privations ${ }^{15}$. Par ailleurs, marginaliser les femmes dans le processus de la construction de la paix ou s'obstiner à ne pas accorder aux femmes une égale participation et une pleine implication dans le processus ne contribue ni à la justice ni au développement ${ }^{16}$. Parler développement sans s'engager dans le

\footnotetext{
${ }^{13}$ Cf. Nadine Puecguirbal, "Women and Children, deconstructing a paradigm". In Seton Hall Journal of Diplomacy and International Relations, Women and Children, Volume V. No 1. Winter Spring 2004, p.12.

${ }^{14}$ Austrian Development Co-operation, Ethiopia Subprogramm Gender \& Democracy 20042006, cité par F. M'boge \& S. Gbaydee Doe, op.cit., p.85.

${ }^{15}$ Ibid., pp.86-87.

${ }^{16}$ Déclaration de Noelen Heyzer parlant de la contribution des fonds des Nations Unies et de l'exécution du $1^{\text {er }}$ débat du Conseil de Sécurité et de landmark Résolution 1325 au sujet des femmes, de la paix et de la sécurité en 2000, cité par M. Elizabeth King. What Difference does It Make? Gender as A Tool in Peacebuilding, p.38.
} 
processus d'intégration du genre c'est naviguer à contre-courant. C'est ce que nous comptons vérifier au regard d'un état des lieux de la parité dans certains pays africains.

\section{ELECTIONS 2011 EN RDCONGO : ENJEUX OU IMPASSE DU PROCESSUS D'INTEGRATION DES FEMMES?}

Avant de faire un état des lieux du processus électoral en RDCongo, nous pouvons indiquer jeter un coup d'œil sur la situation de la parité dans certains pays africains. Nous devons, bien entendu, saluer les efforts fournis par certains Gouvernements africains qui ont atteint un niveau de performance non négligeable de la parité dans les Parlements. C'est le cas du Gouvernement Rwandais qui est en tête ; sur 80 sièges, il y a 39 femmes, soit 48,8\%\%. Ce pourcentage ne concerne que les femmes au Parlement. Lorsqu'on y ajoute le nombre des femmes qui sont membres du Sénat, le Rwanda, semble-t-il atteint un pourcentage de $56 \%$; ce qui le place au premier rang au niveau mondia ${ }^{17} 1$. La Mozambique, sur 250 sièges, 87 femmes, soit 34,8. L'Afrique du Sud, sur 400 sièges, il y a 132 femmes, soit 33\%. Uganda, sur 332 sièges, il y a 102 femmes, soit 30,7\%. Le Burundi, sur 118 sièges, il y a 36 femmes, soit $30,5 \%$. La Tanzanie, sur 78 sièges, il y a 21 femmes, soit 26,9\% et enfin le Lesotho, sur 120 sièges, il y a 30 femmes, soit $25 \%$. Alors que les autres Etats africains ont fourni un très grand effort en atteignant plus ou aux environs de 30\%, la RDCongo traine encore les pas et se retrouve en $42^{\text {ème }}$ position. Sur 500 sièges au Parlement, il n'y a que 42 femmes, soit un faible pourcentage de $8,4 \%$.

La sous-représentativité des femmes dans les institutions congolaises de gestion de la vie publique n'est plus à démontrer. Une étude diachronique faite par plusieurs associations des femmes congolaises a fait un état des lieux de la condition de la femme dans le contexte historique congolais, à savoir dans la société traditionnelle, pendant la période coloniale, pendant la première et la deuxième république, présentement dans la troisième république ${ }^{18} \mathrm{de}$ la RDC. Les différents efforts de mobilisation et de plaidoyer des organisations féminines en RDCongo ont donné comme résultat certaines dispositions de la nouvelle Constitution. En effet, la Constitution congolaise du 18 Février 2006 consacre dans ses articles 12 et 14 les principes d'égalité de droits, de chances et de sexes et disposent de la manière suivante :

Article 12 : «Tous les congolais sont égaux devant la Loi et ont droit à une égale protection des lois ».

Article 14 : «Les pouvoirs publics veillent à l'élimination de toute discrimination à l'égard de la femme et assurent la protection et la promotion de ses droits. Ils prennent, dans tous les domaines civil, politique, économique, social et culturel, toutes les mesures appropriées pour assurer le total épanouissement et la pleine participation de la femme au développement de la Nation. Ils prennent des mesures pour lutter contre toute forme de violences faites à la femme dans la vie publique et dans la vie privée. La femme a droit à une représentation équitable au

\footnotetext{
${ }^{17}$ Je détiens cette information de la Conférence à laquelle j'ai présenté l'essentiel de cette communication en date du 25 mai 2011 à Kigali.

${ }^{18}$ Pour plus de détails, cf Genre et Parité en RDCongo : des paroles aux actes, juin 2010, pp.8-19.
} 
sein de la parité homme-femme dans lesdites institutions. La loi fixe les modalités d'application de ces droits ».

Toutes les options émises sont fondamentales et conformes aux instruments juridiques internationaux, régionaux et sous-régionaux ratifiés par la RDCongo. Il s'agit notamment de la Déclaration universelle des droits de l'homme du 10 décembre 1948, de la Charte de l'Organisation des Nations-Unies, la charte africaine des Droits de l'Homme et des Peuples, la Convention des Nations Unies, le Pacte international relatif aux droits civils, politiques, économiques et sociaux. Tous ces documents militent pour l'élimination de toutes les formes de discrimination à l'égard des femmes. A titre de rappel, le Conseil de Sécurité des Nations Unies avait adopté la Résolution 1325 invitant tous les acteurs de «prendre des mesures apportant un support aux initiatives locales de paix et processus indigènes pour la résolution des conflits et qui impliquent les femmes dans tous les mécanismes des accords d'exécution de paix ». Pour la toute première fois de son histoire, l'ONU reconnaissait la contribution des femmes comme artisans de paix et agents du changement pour la paix. En dépit du fait qu'en plus de ces documents çi-haut cités et la Convention relative aux Droits et du Bien-être de l'Enfant, le Plan d'action de Beijing, la Déclaration de Droits de Vienne, la Déclaration de Philadelphie et le Protocole de la SADC sur le genre et développement proclament tous l'égalité de droits entre les hommes et les femmes et constituent autant d'engagements, la participation des femmes congolaises aux instances de prise de décision aux échelons exécutif et législatif tant au niveau provincial qu'au niveau national démontre que la représentation des femmes est extrêmement très faible.

L'enquête qui a été faite à révélé ce qui suit : au niveau national, l'Assemblée nationale a 43 femmes contre 500 hommes, soit 8,6\%, le Sénat a 6 femmes contre 102 hommes, soit 5,88\% et le Gouvernement National a 5 femmes contre 39 hommes, soit 12,82\%. Par ailleurs, dans les Assemblées provinciales, il y a 9 femmes contre 39 hommes à Kinshasa, soit 23,07\% ; au Bas-Congo, 5 femmes contre 24 hommes, soit 20,8\%; au Bandundu, 3 femmes contre 43 hommes, soit 6,97\%; au Kasai Oriental, (statistiques attendues), au Kasai Occidental, 4 femmes contre 54 hommes, soit 7,4\%; à l'Equateur, 3 femmes contre 105 hommes, soit 2,85\% ; au Katanga, 12 femmes contre 92 hommes, soit 13,02\%; à la Province Orientale, 7 femmes contre 91 hommes, soit 7,69\%; au Sud-Kivu, 3 femmes contre 33 hommes, soit 9,09\%; au Nord-Kivu, 1 femme contre 41 hommes, soit 2,43\%; au Maniema 0 femme contre 24 hommes, soit $0 \%$. Dans les Gouvernements provinciaux de toutes ces Provinces, il y a au Bas-Congo, 1 femme contre 9 hommes, soit 11,1\%, au Bandundu 1 femme contre 9 hommes, soit $11,1 \%$; à Kinshasa, 2 femmes contre 8 hommes, soit $25 \%$; à l'Equateur, 3 femmes contre 7 hommes, soit 42,85\%; au Kasaï Occidental, 2 femmes contre 8 hommes, soit 25\%; au Katanga 1 femme contre 9 hommes, soit 11,1\%; à la Province Orientale, 2 femmes contre 8 hommes, soit 25\%; au Maniema, 2 femmes contre 8 hommes, soit 25\%; au Sud-Kivu, 3 femmes contre 7 hommes, soit 42,85\%; au Nord-Kivu 1 femme contre 9 hommes, soit $11,1 \%$.

Il ressort de ces statistiques que les femmes sont sous-représentées dans le pouvoir législatif au niveau national et provincial parce qu'il n'existe aucune instance qui a atteint $30 \%$. C'est seulement au niveau des Gouvernements provinciaux que deux Provinces se détachent de la 
toile de fond. Il s'agit de la Province du Sud-Kivu et celle de l'Equateur qui ont atteint 42,85\%. Et cela est dû certainement au fait que les deux Gouverneurs de Province ont été sensibles au plaidoyer fait par les organisations féminines au travers de l'Observatoire de la Parité qui est à pied d'œuvre depuis bientôt 3 ans.

La leçon à tirer, c'est que la RDCongo a manqué de prendre des mesures légales et administratives pouvant garantir la jouissance de ces droits par la femme. D'une part, il n'y a pas eu de mesures d'accompagnement des dispositions constitutionnelles. D'autre part, la Loi portant mise en œuvre de la parité homme-femme est une grande première dans l'histoire de la RDCongo. Malheureusement, ladite loi est encore sous examen et ne sera promulguée par le Président de la République qu'à l'issue de son adoption par l'Assemblée Nationale et le Sénat. Le projet de la Loi a comme champ d'application de la parité homme-femme : domaine politique et administratif, domaine économique, domaine social (éducation, santé, famille, travail), domaine culturel, domaine judiciaire et sécuritaire. Cependant, sa grande faiblesse, c'est l'absence des mécanismes et des mesures coercitives faisant de la parité homme-femme une réalité vécue dans l'espace politique, économique, social, culturel, judiciaire et sécuritaire. Il suffit de parcourir cette loi pour se rendre compte qu'elle est parsemée seulement des déclarations d'intention ${ }^{19}$ sans indications des mesures incitatives.

En vue de faire augmenter la participation et la représentation des femmes au sein des instances comme le Parlement, le Sénat, le Gouvernement et autres secteurs de la vie politique, économique, administrative et sociale, il y a lieu d'aller au-delà des déclarations. Il faut imaginer des mécanismes opposables à tous sans exclusive et des sanctions à l'endroit de ceux-là qui osent enfreindre la loi.

\section{QUE FAIRE POUR PROMOUVOIR L'EGALITE DU GENRE ?}

Selon l'Observatoire de la Parité en RDCongo, l'un des mécanismes à utiliser pour élever le niveau de représentation des femmes à des postes de responsabilité, c'est l'introduction, mieux l'imposition du système des quotas dans la Constitution, dans la Loi électorale et les statuts des partis politiques. Ce qui est malheureux, c'est que l'Alliance de la Majorité Présidentielle, pour conserver le pouvoir lors des échéances électorales prochaines, s'est mobilisée pour proposer la révision de la Constitution pour passer de deux à un tour pour

\footnotetext{
${ }^{19}$ II suffit de parcourir quelques articles pour s'en convaincre. Les articles sélectionnés font état des déclarations suivantes : Article 5 : les hommes et les femmes jouissent...; article 6 : les partis politiques sont tenus au respect de la parité...; article 10: l'Etat veille à l'élaboration de la loi électorale (...) l'Etat garantit l'équité dans la participation des femmes et des hommes tout au long du processus électoral, le mode de scrutins et les opérations de vote, particulièrement l'enregistrement des électeurs; article 15: l'Etat prend toutes les mesures nécessaires pour assurer la parité des filles et des garçons en matière de scolarisation (...) l'Etat veille à la prise en charge des enfants orphelins, démunis, filles ou garçons ; article 21 : dans la lutte contre le VIH et SIDA, des mesures spéciales sont prises pour une meilleure protection de la femme, en vue de réduire l'écart du taux de propagation du VIH entre les deux sexes, etc.
} 
l'élection du Président de la République. Il est à craindre qu'une telle option engendre une crise sans précédent après les élections dans le cas où le candidat Président en-tête de la liste ayant atteint 10 à $15 \%$ est proclamé Président de la République. Comment un Président élu avec un tel pourcentage pourra-t-il gouverner un pays dont les plaies dues aux guerres qui ont désorganisé tout le pays et qui ont duré presque deux décennies ne sont pas encore cicatrisées ? Les alliés ont oublié de prendre des mesures incitatives dans leur bataille en faveur de la révision de la Constitution et la loi électorale n'a pas introduit explicitement le système de quotas de $30 \%$ à attribuer aux femmes à tous les niveaux de prise de décision, pourtant l'électorat congolais est essentiellement féminin.

En vue de rendre la représentation des femmes opérationnelle au niveau du Parlement, les organisations féminines militent pour un système de liste zébrée ou bloquée, c'est-à-dire composée alternativement d'un homme et d'une femme ${ }^{20}$. Si donc cela ne figure pas dans la Loi électorale pour les élections prévues pour Novembre 2011, il y a donc impasse. Etant donné que la RDCongo a ratifié les instruments juridiques sous-régionaux, régionaux et internationaux, il est indispensable que les gouvernants poussent cette logique jusqu'au bout par l'application de tous ces instruments dans tous les espaces de la vie nationale. Les femmes et les organisations féminines ne devraient pas croiser les bras mais continuer à organiser un plaidoyer fort, persuasif et dissuasif à la fois pour que dans les cinq ans à venir la Constitution indique clairement ce pourcentage d'au moins $30 \%$ à attribuer aux femmes dans tous les espaces de décision et que la loi électorale tire les conséquence de cette disposition constitutionnelle.

On peut facilement vérifier qu'au Rwanda (art 11 et 16), au Burundi (art. 13, 22) comme en RDC (art. 13), non seulement la Constitution interdit toute forme de discrimination, qu'elle résulte de la loi ou d'un acte de l'exécutif ; mais elle exige, par ailleurs aux pouvoirs publics, qu'Ils prennent, dans tous les domaines, notamment dans les domaines civil, politique, économique, social et culturel, toutes les mesures appropriées pour assurer le total épanouissement et la pleine participation de la femme au développement de la nation.

Ainsi au Rwanda, l'Etat s'est engagé à garantir «l'égalité entre les femmes et les hommes reflétée par l'attribution d'au moins trente pour cent des postes aux femmes dans les instances de prise de décision » $\left(\right.$ art. $\left.9-4^{\circ}\right)$. Ce qui s'observe déjà au sein de l'Assemblée nationale où les femmes sont représentées à un peu moins de 50\%. L'exemple du Rwanda avec un pourcentage de 48,8\% de représentation des femmes au Parlement est impressionnant.

En RDC, la Constitution précise que «La femme a droit à une représentation équitable au sein des institutions nationales, provinciales et locales ». Et que «L'Etat garantit la mise en œuvre de la parité homme-femme dans lesdites institutions » (art. 14). Toutes ces déclarations n'ont pas force de loi car les nominations qui sont faites ne reflètent en rien le respect de ces dispositions. L'effort qui reste à fournir est d'arriver à imposer la représentation des femmes au moyen du système de quotas à $30 \%$ au niveau du Parlement et du Gouvernement et même des entités décentralisées. L'introduction des quotas n'est pas du tout ex nihilo parce que,

\footnotetext{
${ }^{20}$ Pour plus de détails, consulter Rapport sur l'état de la parité en RDCongo élaboré par l'Observatoire de la Parité en RDCongo, 2010, pp.1-49.
} 
comme l'a si bien dit Sumie Nakaya, l'égalité du genre dans la construction de la paix postconflit dépend (1) du cadre institutionnel de la gouvernance post-conflit incluant les arrangements du partage de pouvoir ; (2) de la base structurelle des relations du pouvoir en société, tels que la classe, le clan et autres formes du fait d'être membres du groupe à partir duquel la jonction du genre ne peut être construite indépendamment; (3) le support international à l'intégration du genre dans la construction de la paix. L'incorporation de 30\% de quotas pour les femmes dans les accords de paix est une partie du cadre institutionnel pendant que la base structurelle du pouvoir continue de dicter les relations du genre dans les maisons, les communautés et le processus de prendre des décisions dans plusieurs de sociétés post-guerre $^{21}$. On peut donc noter que le système des quotas fait partie intégrante du cadre institutionnel découlant de l'agenda international encourageant le partage du pouvoir dans la dynamique post-conflit de la construction de la paix et de développement durable.

A mon humble avis, pour éviter que le système de quotas imposés d'en-haut n'aboutisse à une représentation artificielle des femmes sans soutien à la base, toute la démarche doit être soustendue par un travail de grande envergure, celui de l'éducation de toutes les couches des populations dans tous les secteurs (écoles, universités, églises, groupes associatifs, familles, etc). La stabilité du pays et même de la Région en dépend.

Dans ce programme d'éducation, il est important que les femmes cessent d'être considérées comme des victimes appartenant à des groupes vulnérables. En vue de corriger dans la durée la vision du monde selon une norme de référence masculine, deux démarches peuvent être envisagées : (1) l'action éducative qui est tridimensionnelle, c'est-à-dire d'une dimension intellectuelle, culturelle et politique et (2) entreprendre des réformes structurelles qui promeuvent l'égalité intra-groupes (hommes et femmes) et l'égalité inter-groupes (représentation à égalité des femmes Tutsi et femmes Hutu et assurer une représentation nondiscriminatoire dans les Etats où il y a plusieurs communautés). L'intégration de la perspective du genre dans le processus requiert l'évaluation des implications des hommes et des femmes à toute action planifiée, législation, politiques ou programmes inclus, dans toute ère géographique et à tous les niveaux ${ }^{22}$ c'est autant dire que l'action affirmative dans les réformes institutionnelles allant dans le sens de promouvoir l'égalité du genre dans la prise de décision ne pourra commencer à porter les fruits que si les femmes occupent des positions à tous les niveaux de prise de décision (dans le processus de construction de la paix en période post-conflit) et qu'elles sont assises à la table de négociation à égalité de voix avec les hommes (dans le processus de négociation de la paix).

La principale tache de la construction de la paix à partir d'une perspective de genre de contribuer à la transformation des mentalités au moyen de l'éducation et de mener à bon port les réformes structurelles qui promeuvent l'égalité intra- et inter-group au niveau institutionnel et à l'intérieur des relations de pouvoir.

\footnotetext{
${ }^{21}$ Sumie Nakaya, Women and Gender Equality in Peacebuilding. In: Journal of Peace Education, p.147.

${ }^{22}$ Cette définition a été adoptée par le Conseil Economique et Social de l'ONU, E.1997.L.10, 17 juillet 1997, paragraphe 4.
} 


\section{CONCLUSION}

Les stéréotypes et les perceptions différenciées des rôles sociaux que peuvent jouer hommes et femmes dans la société impliquent que les femmes qui jouissent d'un mandat politique sortent du lot. Il se fait que les femmes sont sous-représentées au niveau du pouvoir législatif et du pouvoir exécutif tant aux instances nationales que provinciales. Il en est de même dans les services de l'administration publique (bourgmestres, maires des villes, magistrats, juges, police etc). Des enquêtes ont établi que les femmes non seulement elles sont sous représentées dans les instances de décision mais aussi elles sont victimes des maltraitances au niveau du toit conjugal. Elles subissent aussi plusieurs formes des violences graves et d'abus sexuels. Dans les milieux scolaires, universitaires et professionnels, les filles et les femmes sont victimes de harcèlements sexuels, de viols et d'abus portés vers le phénomène des PST (points sexuellement transmissibles).

Pour mettre en branle l'égalité du genre, il convient de s'empêcher de développer les stéréotypes relatifs à la division sexiste du travail qui consiste à allouer des taches et responsabilités aux mâles et femelles en accordant plus d'importance et valeur à celles assignées aux hommes qu'aux femmes, non seulement à la maison mais aussi dans les milieux professionnels et dans la société en général. L'objectif n'est pas d'annihiler les différences qui existent entre les deux sexes mais de donner aussi bien aux hommes qu'aux femmes les mêmes opportunités pour la réussite des objectifs importants dans la société, notamment la qualité de la vie, l'éducation, l'emploi et le revenu. Or la qualité de la vie peut être affectée par les discriminations dues à la race et l'ethnicité, la classe et le statut économique, la capacité et la sexualité ${ }^{23}$. Ces politiques fixistes n'encourageant pas l'égalité du genre sont des anti-valeurs à combattre étant donné qu'elles limitent les potentialités et les chances de la vie d'un grand nombre de personnes dans nos sociétés et dans le monde. La perception négativiste et fixiste des femmes fait beaucoup de tort à nos Etats.

La stratégie conduisant au développement implique l'émergence d'un autre type de leaders. Le leadership féminin dans la consolidation et la durabilité de la paix au niveau de la communauté a prouvé être essentiel dans la construction de la Nation. C'est ce que dit l'ambassadrice Gertrude Mongela en ces termes :

The participation of women not only provides equal opportunity on a practical level, but also offers a new perspective and diversity of contributions to policy-making and priorities for development. Addressing gender issues is an important strategy in stimulating development, in alleviating poverty, and strengthening good governance. The main reason for this is that development can only be sustainable where its material benefits are fairly distributed, especially to those most in need, most disadvantaged and most vulnerable - and these people are often women ${ }^{24}$.

\footnotetext{
${ }^{23}$ Rhoda Reddock. Gender-equality, Pan-Africanism and diaspora. In: International Journal of African Renaissance Studies. Multi - Inter - and Transdisciplinarity. Vol 2, № 1, Octobre 2007, pp.256-257.

${ }_{24}$ Gertrude Mongela, "Women take on the old boys club". Action for Southern Africa

(ACTSA), April2004. Cf. http://www.actsa/org/News/010504 democracy.htm.
} 
Nous pouvons conclure que la participation des femmes non seulement fournit une opportunité égale sur le plan pratique mais aussi offre une nouvelle perspective et diversifie les contributions dans le cadre du développement. La participation des femmes dans les affaires politiques est plus que jamais une voie obligée pour les pays en voie de développement. La question de l'égalité du genre est une stratégie capable de stimuler le développement, réduire la pauvreté et renforcer la bonne gouvernance. Et cela passe par impliquer les groupes les plus vulnérables, les plus désavantagés ou qui sont dans le besoin, j'ai cité les femmes. Les questions concernant comment le budget national est partagé dans tous les secteurs de l'économie et comment les besoins sont abordés en utilisant le budget national, toutes ces questions deviennent pertinentes pour garantir la paix à long terme au niveau national, régional et global. Les inégalités en termes d'accès au revenu aussi bien pour les femmes que pour les hommes, le manque d'accès au contrôle et à la production des ressources contribuent à la perpétuation du conflit. 